TITLE PAGE

1. The title of the article:

\title{
A 3D MACHINE VISION METHOD FOR NON-INVASIVE ASSESSMENT OF RESPIRATORY FUNCTION
}

2. The name and initials of each author:

L. N. Smith, M. L. Smith, M. E. Fletcher and A. J. Henderson

3. The department and institution to which the work should be attributed:

Department of Engineering, Design and Mathematics, University of the West of England, Bristol.

4. The name, postal and email address, and telephone number of the author responsible for correspondence and to whom requests for reprints should be addressed:

Professor Lyndon Neal Smith

Professor in Computer Simulation and Machine Vision

Department of Engineering, Design and Mathematics, T Block

University of the West of England, Bristol

Bristol BS16 1QY UK

Email: Lyndon.Smith@uwe.ac.uk

Tel. +44 (0)1173282009

5. Sources of financial support:

This paper presents independent research funded by the National Institute for Health Research (NIHR) Invention for Innovation (i4i) Programme (II-FS-0908-10078); and the University of the West of England, Bristol.

6. Category in which the manuscript is being submitted:

Original Article

7. Word count and number of figures:

Words: 5,234. Figures: 7.

There are no financial and/or personal relationships that might bias the authors' work and the authors have conflicts of interests in relation to this manuscript. No potential conflicts exist. 
The paper describes original research that has not been reported elsewhere, has not been simultaneously submitted elsewhere and has not been accepted for publication anywhere else. The manuscript has also been approved by each of the co-authors; and we have permission to publish, from the NIHR. The project received ethical approval from the Ethics Committee at the University of the West of England, Bristol. 


\section{ABSTRACT \\ Background}

Respiratory function testing is important for detecting and monitoring illness, however it is difficult for some patients, such as the young and severely ill, to perform conventional tests that require co-operation and/or patient contact.

\section{Method}

A new method was developed for non-contact breathing measurement, employing photometric stereo to capture the surface topography of the torso of an unconstrained subject. The surface is integrated to calculate time-dependent volume changes during respiration.

\section{Results}

The method provides a useful means of continuously measuring volume changes during respiration with high spatial and temporal resolution. The system (NORM) was tested by comparison with pneumotachometry equipment and a clear periodic signal, of a frequency corresponding to the reference data, was observed.

\section{Conclusion}

NORM is unique in performing breathing monitoring (with potential diagnostic capability) for unconstrained patients in virtually any lighting conditions (including darkness during sleep) and in a non-contact, unobtrusive (i.e. using imperceptible light) fashion. 


\section{INTRODUCTION}

Respiratory function testing is a useful method for monitoring the progression of clinical conditions, and is an important marker of serious illness. The utility of applying non-contact breathing measurement technology is well illustrated in the case of infants. Studies have demonstrated that lung function soon after birth is associated with development of asthma later in childhood, $(1,2)$. Analysis of respiration in early life can also provide indicators of later lung disease such as chronic obstructive pulmonary disease; although more research is needed to define the nature of the relationships involved. The advantage of the technology presented in this paper is that it could be applied in large, community-based samples to measure aspects of early lung function that might indicate future disease in screening/monitoring programs. In conditions such as cystic fibrosis (CF) lung function is impaired prior to diagnosis, even in early infancy (3). Thus reduced lung function detected in screening could facilitate the identification of children with CF before the disease presents with symptoms. Also, patients with severe chest wall deformities, such as those associated with scoliosis, usually suffer breathing impairment, where respiratory pattern may change in association with progression of disease; and this can be difficult to detect using simple tests such as counting respiratory rate. Non-contact respiratory measurement can also offer a means of monitoring the effects of corrective orthopaedic surgical interventions (4). For example, when scoliosis is associated with muscle disease perioperative respiration can be critical, but patients often have difficulty with standard blowing tests. A number of studies have also found that breathing characteristics can provide a means of predicting cardiac problems or requirement for intensive care (5-8). These studies have found respiratory rates to be more reliable indicators of serious clinical events than other measurements such as blood pressure or pulse (6); it has been reported that alterations in respiratory rate can be 
used to identify patients at high risk with a specificity of $95 \%$ (8). However, while there is increasing evidence that lung development during early life is important for respiratory health through the life-course, some patients, particularly the very young, are not able to perform the existing tests. Current procedures are conducted by "contact" test methods, many of which become impossible to use with young children or patients who are either too young or too ill to cooperate. There are no tests available that require the patient to do no more than simply sit without a face mask, special chest bands/markers or a mouthpiece and undergo continuous capture of the whole respiratory system function. Consequently it is not currently possible to detect minute falls in ventilation due to progressive respiratory or cardiac or neurological disease. Therefore, for significant patient numbers across all ages, no appropriate assessment tool exists, even though continuous monitoring could provide early warning of disease, that repeating conventional tests does not offer (and that simple tests such as respiratory rate might miss). This deficiency highlights the urgent need for research into clinical monitoring and diagnosis for enabling respiratory function testing in both specialist centres and primary care. To illustrate the limitations of current technologies employed clinically, a brief review is given here of existing techniques for respiratory function testing.

Methods for monitoring respiratory function can be classified into two types: contact and noncontact. In the former case sensors are placed in contact with the patient's body in order to detect parameters that can be used to measure breathing. These parameters include: airflow, total thoracic/abdominal movement, $\mathrm{CO}_{2}$ and $\mathrm{SpO}_{2}$ based methods, acoustics and ECG derived respiration. There is not enough space here to describe the research that has been undertaken on all of these techniques. However, it is worth noting that they all suffer from the disadvantage of 
requiring some form of sensor to be applied to the patient. This can cause discomfort, may mean they are not suitable for use during sleep, can lead to errors due to distress caused by the contact device and may of themselves affect the parameters of interest. For example, measuring flow by a pneumotachograph at the mouth and/or nose requires contact with the patient's face, which may alter the breathing pattern e.g. by the re-breathing of exhaled air (known as dead-space). Also, in some cases such as young children or the critically ill, patient cooperation often cannot be attained so that it is not possible to employ contact methods. However, a number of contact methods are commonly employed and are therefore worth mentioning in more detail; specifically spirometry, inductive plethysmography and airflow based methods. A spirometer is a device for measuring the volume of air inspired and expired by the lungs. It detects the flow rate of gases by measuring pressure differences across a fine mesh; and then flow is integrated to produce a pot of volume against time. Incentive spirometry can be used in children as young as 3 years, but this leaves a significant gap in measures during the most rapid phase of postnatal lung development. Spirometers are usually designed to measure lung capacity rather than to monitor normal tidal breathing; and commonly employ the simple method of total integrated air volume. For this work a spirometer was calibrated using the continuum of airflows exhibited in tidal breathing, thereby providing precision ground-truth data. Inductive plethysmography (IP) employs a number of insulated coils of wire that are sewn into bands and then positioned around the chest and abdomen. The electrical induction of the coils is monitored and used to measure the change in cross-sectional area of the rib cage where the bands are located. This sensing technique is commonly employed and has been found to produce breathing measurements that correlate well with lung volume measurements determined by pressure variations in a control volume (9). Disadvantages of the technique include the fact that it requires patient contact (so may not be 
suitable for use during sleep) and potential technological limitations such as electrical interference with other equipment (9). Also because inductance varies with length (circumference) and babies have compliant chest walls (change shape readily depending on position), movement from one position to another can invalidate the calibration. Finally, the very limited spatial resolution of IP limits its utility as a diagnostic tool, since the ability to measure movement and breathing at locations between the bands may assist with clinical assessments. For example, high-spatial resolution measurement of respiratory muscle dysfunction could be useful since it has been found that this condition often goes undetected until it becomes more severe (10). Airflow methods make use of the difference in, for example, the temperature of exhaled and inhaled air; where these differences can be used to infer respiratory rate. These types of systems therefore require a sensor to be attached to the airways, such as a thermistor to measure the temperature of the inhaled and exhaled air. Disadvantages include the obtrusive nature of the sensor and accuracy limitations, such as the calibration issues associated with the non-linear signal from the thermistor. Another more accurate approach is to employ a nasal pressure transducer, however the transducer can be uncomfortable and can itself affect breathing. For very young children, or patients with significant respiratory compromise or fatigue related disorders (e.g. muscular dystrophies) use of such transducers or effort dependent procedures requiring coordination and cooperation can be challenging if not impossible, preventing accurate monitoring of the disease process. Therefore existing contact sensor systems often rely on methodologies prone to significant error (11) and/or limited resolution.

Non-contact methods for respiratory monitoring include the following: optical (including thermal imaging and laser displacement measurement) sensors, CT scanning, and radar and thermal 
sensor systems. Again, it is not possible to enter here into a detailed review of all the non-contact techniques reported in the literature. Instead selected previous work will be mentioned to illustrate the type of research being undertaken and its relevance to the authors' work. One of the most commonly researched non-contact methods is that of optical breathing monitoring, where the patient is illuminated by one or more light sources (often employing structured light such as stripes) and the resulting images of the patient are captured using a camera. If images are captured continuously then changes in the illumination pattern can be used to calculate body shape changes and hence breathing volumes. Early research employing triangulation of light stripes indicated that good accuracy could be obtained (12), but at the time the technique was not developed into systems that could be used on the ward, presumably due to the expense and performance limitations associated with the imaging and computing technology available. Today, high-resolution digital cameras and high-performance computing hardware are both relatively low cost, so that optical systems for real-time breathing monitoring are becoming a viable commercial possibility. Systems that are available on the market tend to employ triangulation, either through use of reflective markers that are placed at selected positions on the body (13), or by projecting a pattern of coloured light onto the chest and abdomen (14). Although these systems claim good accuracy, both are associated with disadvantages, which are principally related to the degree of structuring of the environment that is required. In the case of the marker system, since the markers need to come into contact with the patient it is not strictly a non-contact approach. Also, the markers can be complicated and tedious to place and errors in placement as small as $1 \mathrm{~mm}$ can result in the marker not being recognized by the device (13). There are also practical issues associated with the marker system: the purchase and set-up costs are high, the subject needs to stand within the machine which is restrictive, and they need to be 
bare-chested for marker placement and data acquisition - some subjects may be uncomfortable with this and same-sex technicians may be required, thereby further increasing costs. The patterned light system suffers from a similar disadvantage in that the patient needs to be in a specified position - for example, in a chair with the pattern projection equipment mounted in front of them. Since the method employs a data projector for producing the patterns of light there is also the need for the patient to be located at a set distance from the projector for the pattern to be in focus; and there is a danger of the pattern going out of focus towards the side of the trunk where the skin is further from the projector than on, say, the front of the trunk. A further limitation is that the system employs visible light; and this in combination with the positional constraints involved would be expected to make the system unsuitable for employment with sleeping patients. This is quite important, since detection of sleeping related disorders, such as Obstructive Sleep Apnoea, could be an important and valuable potential application of any noncontact breathing measurement system. Other reported research on non-contact breathing measurement has involved use of laser Doppler vibrometry (15), laser displacement techniques (9) and thermal imaging where the temperature of the skin is used as a measure of breathing (16). These three approaches all suffer from the limitation of not offering high spatial resolution; i.e. not being able to measure the body shape changes simultaneously at various points, as is useful for diagnostic purposes. Therefore there is currently no non-invasive (or obtrusive/constraining/disruptive) highly accurate system requiring minimal cooperation to monitor or assess respiratory function applicable to all ages; thereby impeding studies of directly comparable (as opposed to directly related) data from childhood to adulthood $(3,17)$. 
This paper describes work that was undertaken to enable development of a novel, non-invasive sensing method for non-contact assessment of respiratory muscle function by monitoring changes in the three-dimensional (3D) surface details of the human trunk in real time. The optical system developed employs a technique that has been designated as 'Novel Non-invasive Assessment of Respiratory Function' (NORM). Here an infra-red version of a vision technique known as 'dynamic photometric stereo' captures and tracks motion details of the chest and abdomen walls, recording 3D shape and dimensional variation of the body dynamically during breathing. We have conducted in-vivo experiments using the NORM system, to demonstrate proof of principle of measurement of volume-time signals for tidal breathing of volunteers. We have also begun work on in-vivo regional differences in chest wall motion that can be detected using the NORM system. 


\section{MATERIALS AND METHODS}

The NORM prototype system was developed in the UWE Machine Vision Laboratory, to demonstrate the use of machine vision technology for non-invasive and non-restrictive monitoring of patients' breathing. Extensive system development was required in areas that included mechanics, software, electronics, physiology, optics, imaging, pneumatics and ergonomics. The first NORM proof of principle prototype is shown in Figure 1, where the various system components are visible, including the patient support that allows him or her to recline comfortably. Patients with breathing difficulties can breathe more easily by sitting more upright, with gravity assisting the diaphragm. The framework allows flexible positioning of lighting and cameras. A mannequin was employed for system development, and then replaced with adult volunteers for capturing breathing data. 


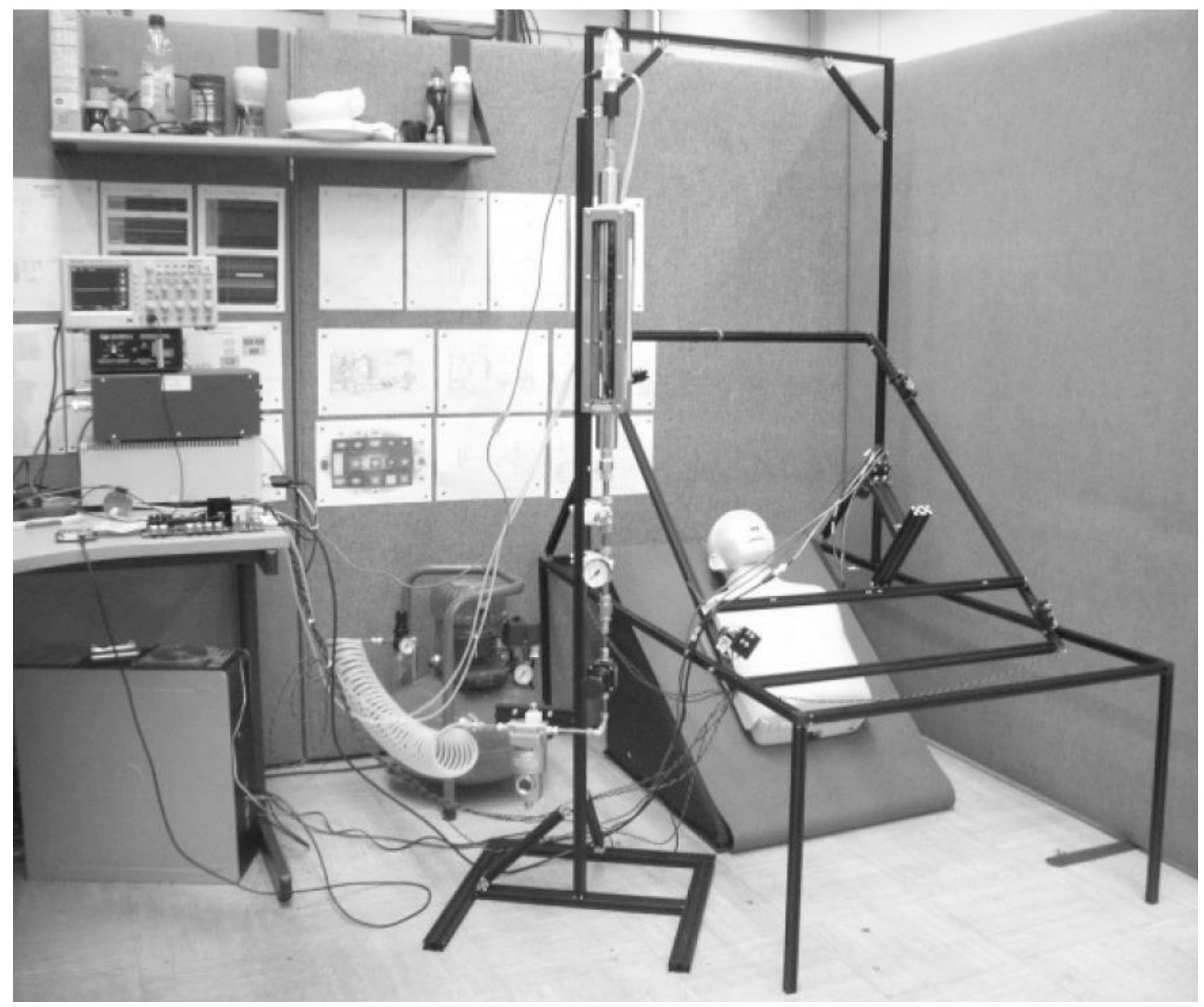

Figure 1. NORM Proof of Principle System; the final clinical system will either be mounted on the wall/ceiling, or will employ a simple bedside type stand.

NORM employs a conventional PC with a high capacity hard drive (1TB) that facilitates the storage of over 12 hours of continuously acquired high resolution images, enabling the monitoring of breathing conditions for sleeping patients. These images were demonstrated to capture time-stamped images for over 10 minutes without dropping a frame, and with a $<2 \mu \mathrm{s}$ timing precision. Lighting and image capture are synchronised by the NORM system control and 
interface electronics. The spirometer (pneumotachometry equipment), visible near the top of Figure 1, was calibrated for tidal breathing by employing an accompanying pneumatic rig prior to the capture of spirometric data. This provided ground truth breathing data with which to compare the NORM optical data, for benchmarking NORM.

In addition to providing overall breathing volume measurement, the NORM system provides high spatial resolution for localised diagnoses of lung conditions, while using an affordable digital camera. Good quality image capture was achieved by employing an area scan monochrome industrial GigE camera, which provided a resolution of 1.4 million pixels at 75 frames per second, and a dynamic range extending into the infrared. This latter characteristic was particularly important in the NORM application, since long-wavelength light is employed for illumination of the patients. Illuminating the patient using wavelengths beyond the visible spectrum facilitates system operation in the dark, which is critical for use of the system for sleep studies. Also, employing invisible wavelengths means that the system will not disturb the patient and other surrounding persons. This is beneficial since the vision technique employed involves flashing light sources which would cause disturbance if visible, or might be inadvisable for use with epilepsy sufferers. Therefore an illumination of wavelength 850nm was employed. 


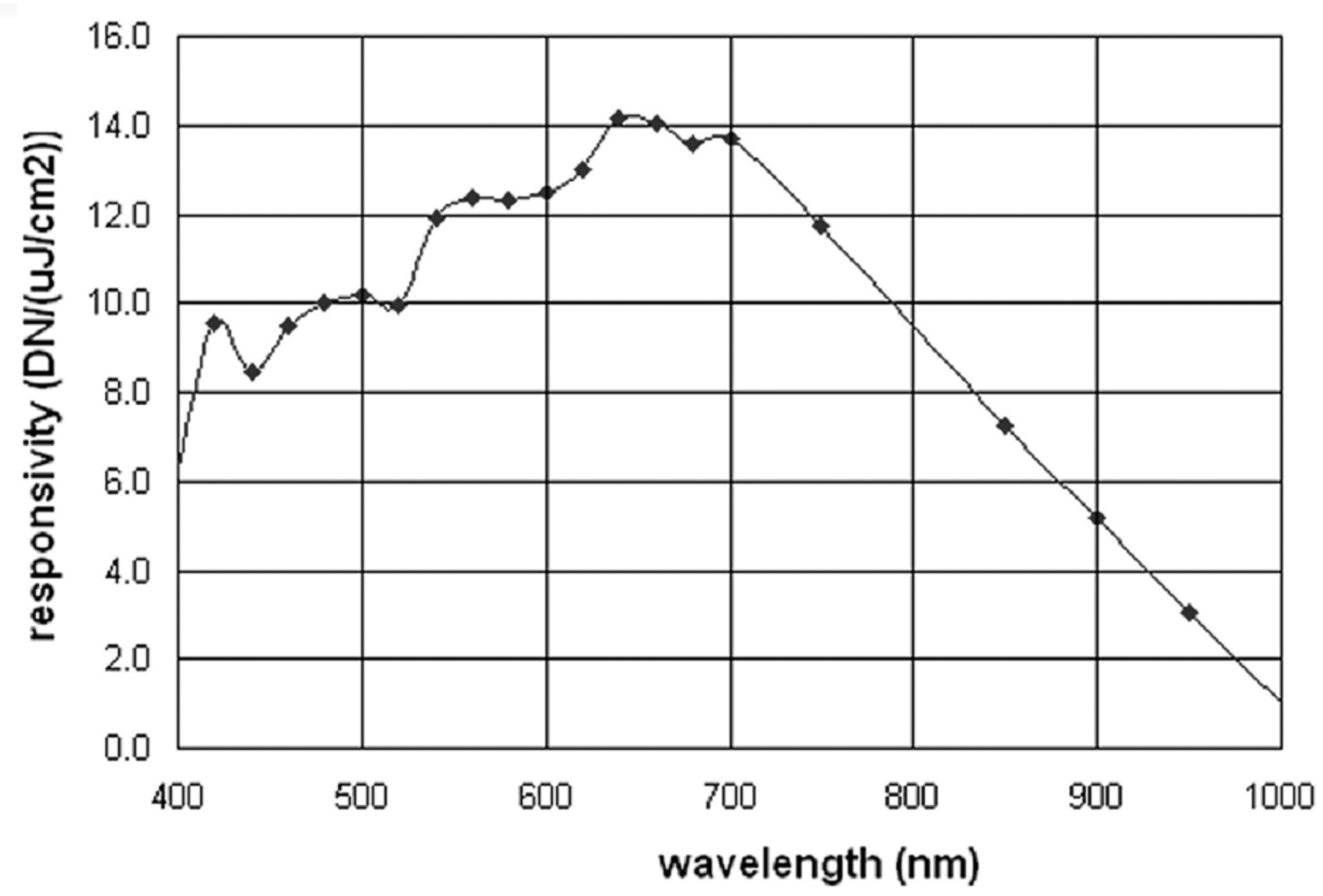

Figure 2. Spectral response of Genie HM1400 / XDR Area Scan Monochrome Camera (Teledyne DALSA).

Figure 2 shows the spectral response of the camera employed, and although at longer wavelengths it drops considerably from its peak at $650 \mathrm{~nm}$, it can be seen that even at the relatively long wavelength of $850 \mathrm{~nm}$ the sensitivity is reasonable at around $7 \mu \mathrm{J} / \mathrm{cm}^{2}$. It was also necessary to employ a lens optimised for transmission at the wavelength used, therefore a compact high resolution lens was employed (Schneider C-mount Cinegon), which is designed for use in the range $400-1000 \mathrm{~nm}$. The light sources themselves comprised modern high luminosity light emitting diodes (OSRAM SFH 4730), which feature the high output optical power of 3 watts, a black frame to minimise scattered light and an absence of optical focusing resulting in a 
relatively uniform and wide illumination characteristic. The emission spectrum of these diodes is shown in Figure 3.

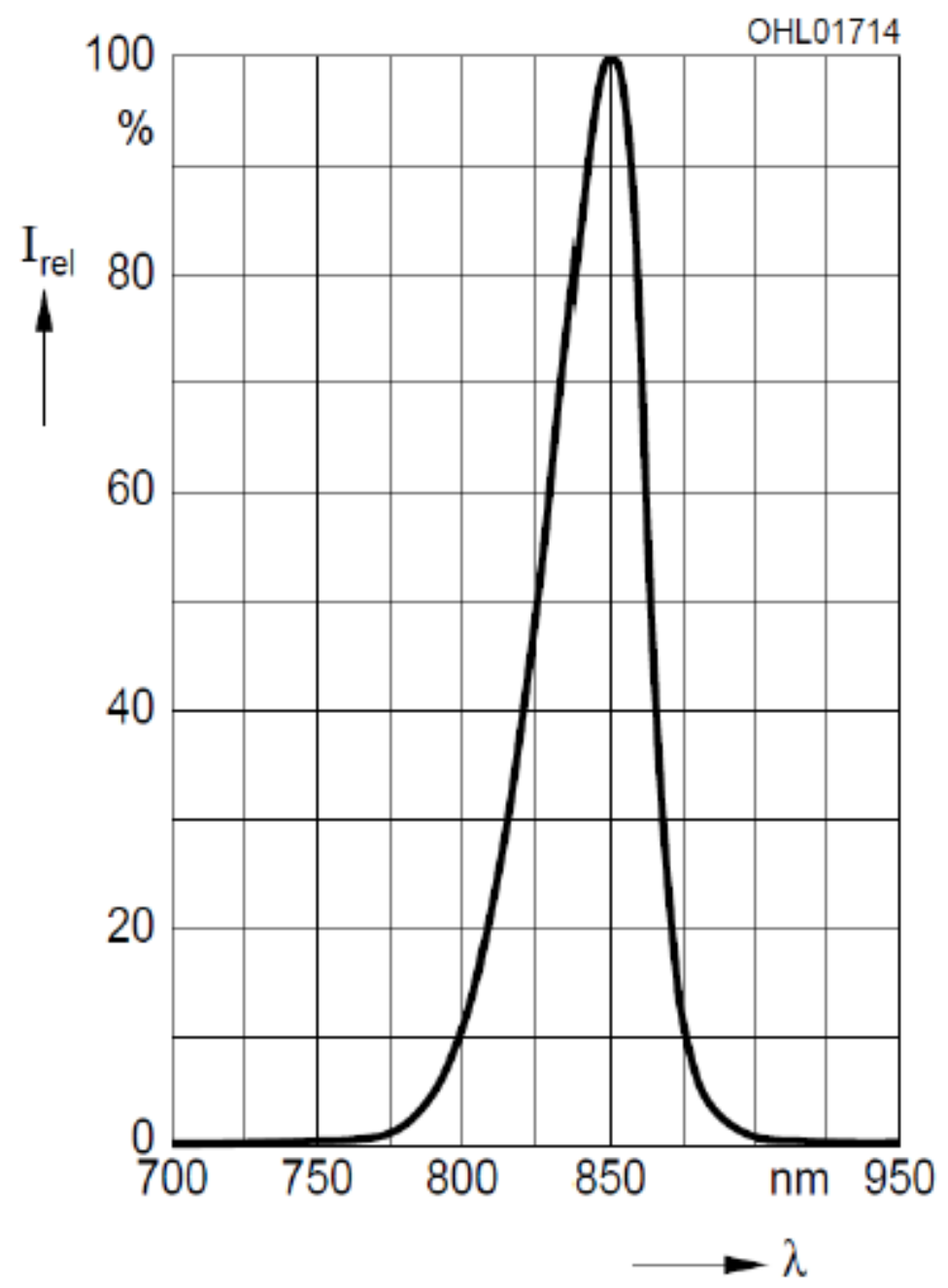

Figure 3: Emission spectrum of SFH 4730 Infrared LEDs.

Employing a wavelength longer than that of visible light provides the additional benefit of enabling optical filtering to minimise interference from ambient mains powered fluorescent lighting. In determining the preferred optical filter characteristics, it is necessary to ensure good 
transmission at the LED peak wavelength while minimising transmission at other wavelengths. This results in the signal to noise ratio being maintained/enhanced, so that camera integration times do not need to be increased to an extent that would impact the image acquisition rate, which is crucial for maximising the temporal resolution of the system. Coloured-glass infrared filters were employed since they are cost-effective and insensitive to the angle of incidence. The infra-red filter Schneider SKR FIL 093 was used, which has a transmission profile as shown in Figure 4.

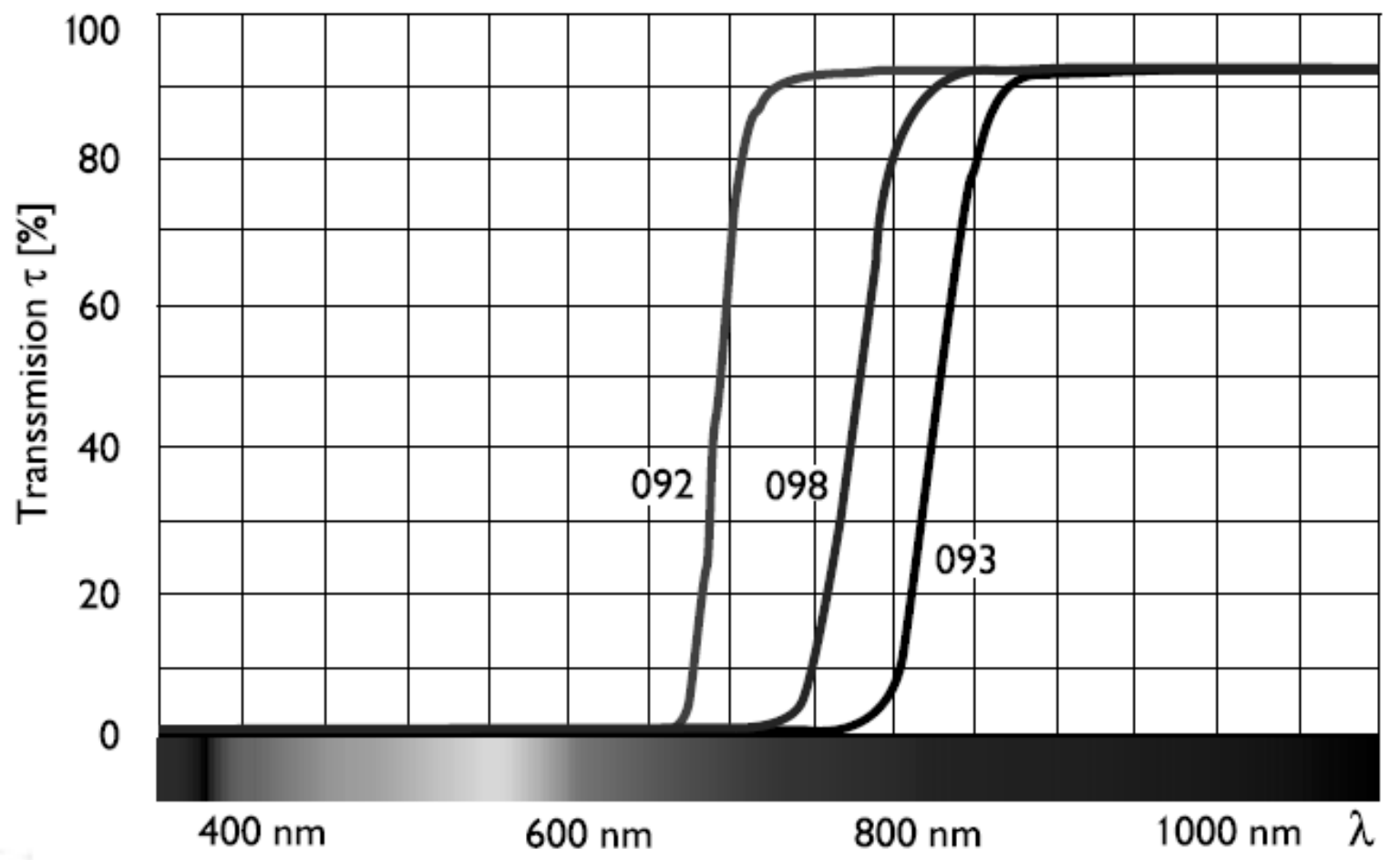

Figure 4. Transmission Profile for Infra-Red filter Schneider SKR FIL 093, (profiles for two other filters are also shown). 
It can be seen that at the wavelength of the LEDs, $850 \mathrm{~nm}$, the transmission of the filter is relatively high. It was found that the filter decreases the signal to $63 \%$, while the noise component comprising wavelengths shorter than $750 \mathrm{~nm}$ are attenuated by the filter to less than $1 \%$ of their original value. Therefore the filtered system will have a signal to noise ratio at least a factor of 60 times better than in the unfiltered case. Clearly therefore, it is worth employing optical filtering in systems of this type.

Using the above components, NORM recovers surface orientation information which is processed to provide a bulk volume measurement of the patient's torso volume over time. Surface recovery is achieved by using a photometric stereo technique that employs multiple light sources, at known locations, and a single camera.

\section{Photometric 4D Approach for Recovery and Modelling of the Torso}

Analysis of the three dimensional topography of the surface of an object can be achieved by illuminating the object from at least three different directions and observing the reflectance at a single viewpoint (18). Energising a single light source and capturing an image (and repeating this for each light source), enables calculation of the following three unknown variables at pixel / sub-pixel resolution, for each surface element: roll-angle, pitch-angle and albedo. (Albedo is a dimensionless quantity quantifying the reflectance of a material from $0 \%$ for a perfect absorber to $100 \%$ for a perfect reflector.) This is the technique known as photometric stereo, which can be used to derive the normal $(\boldsymbol{n})$ at a point on the surface; and since there are three unknowns, images from at least three light sources are required to find the unknown variables. Figure 5 
shows the surface normal vector defined in terms of its three direction cosines, within the lighting coordinate frame $\left(\mathrm{X}_{\mathrm{L}}, \mathrm{Y}_{\mathrm{L}}, \mathrm{Z}_{\mathrm{L}}\right)$.

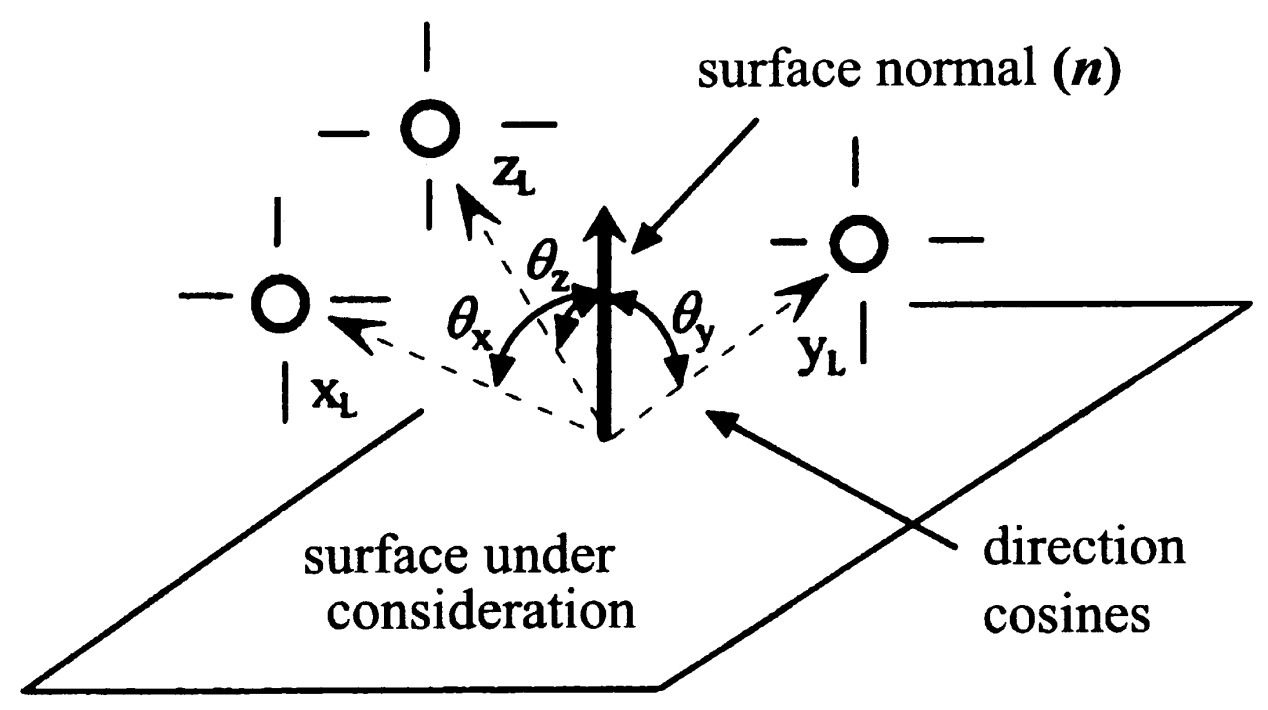

Figure 5. Photometric stereo geometry for determination of the surface normal vector $\boldsymbol{n}$.

The surface of the object is illuminated by at least three light sources at known locations, each with a directional vector, with the camera above and pointing directly towards the surface.

A matt surface will reflect light approximating to Lambertian reflectance, exhibiting a cosine or scalar product relationship with respect to the light vectors. The magnitude of these three vectors (which form the axes of the lighting coordinate frame) will be determined by the intensity of the three respective illuminates, which should be arranged to be equal. The corresponding pixel intensity within each of the three acquired images, one respectively for each light $\left(\mathrm{X}_{\mathrm{L}}, \mathrm{Y}_{\mathrm{L}}, \mathrm{Z}_{\mathrm{L}}\right)$, will be related to the surface normal vector components at the given location. Hence, the three normal vector 
components can be calculated from the three respective images. Once the normal vector components are known, the magnitude of the surface normal vector can also be calculated, and will be directly proportional to the albedo of the surface material at the location being considered. Since the intensity will be given by the dot product of the lighting and surface normal vector, the direction cosines of the normal vector can be expressed as:

$$
\begin{aligned}
& \theta_{x}=\cos ^{-1}\left(\boldsymbol{x}_{L} \cdot \boldsymbol{n} / \boldsymbol{x}_{L}|| \boldsymbol{n} \mid\right) \\
& \theta_{y}=\cos ^{-1}\left(\boldsymbol{y}_{L} \cdot \boldsymbol{n} /\left|\boldsymbol{y}_{L}\right||\boldsymbol{n}|\right) \\
& \theta_{z}=\cos ^{-1}\left(z_{L} \cdot \boldsymbol{n} /\left|z_{L}\right||\boldsymbol{n}|\right)
\end{aligned}
$$

Therefore, photometric stereo can provide 3D information on the local gradient of the surface, as well as the $2 \mathrm{D}$ local surface albedo at a pixel or by using interpolation a sub-pixel level. Once the gradients of the surface over each pixel area of the object are known, it is possible to reconstruct the surface topography using each surface element and linear algebra. Calibration corrections were incorporated to compensate for the assumption of the light sources being at infinity (this improves the accuracy of the lighting vector, by considering the direction as a function of pixel position) and the dispersion of radially emitted light over distance (inverse-square law). Lastly, variations in lighting intensity were corrected by performing irradiance measurements of each light source using an optical power meter and custom made dark box. 
To convert the recovered surfaces into an instantaneous volume measurement, an integration technique is used, which employs the surface elements. Preliminary work has also begun on modelling the vertical cross-section of the torso as vertically stacked ellipses. This enables breathing performance to be evaluated at various localised regions of the torso; thereby potentially providing measurement of Regional Ventilation Inhomogeneity (RVI), in addition to the monitoring of conventional tidal breathing. 


\section{RESULTS}

The NORM electronics and algorithms control the camera and lighting to microsecond precision. Auxiliary data such as spirometer data and environmental data can be captured at rates of kilohertz, surpassing the recommended rate of $200 \mathrm{~Hz}$ for Spirometry acquisition, and enabling detection of fine structure in respiration and lighting harmonics. The data acquisition software enabled easy capture of high resolution image data at a consistent rate of over $50 \mathrm{~Hz}$, enabling a dynamic analysis of $3 \mathrm{D}$ chest movement at $10 \mathrm{~Hz}$ (four frames with directional lighting, and a background frame), which is over an order of magnitude faster than the rate of typical tidal breathing $(\sim 0.2 \mathrm{~Hz})$. The calibration apparatus successfully provided pneumatic flow rates in the range $\pm 3 \mathrm{~L} / \mathrm{s}$, with a high precision of $\pm 7.5 \mathrm{~mL} / \mathrm{s}$. Preliminary results suggested that mains powered fluorescent lighting introduced an optical noise component into the dynamic volumetric data. The fluorescent lighting fundamental was found to be $100.055 \mathrm{~Hz}$, with a FWHM of $0.06 \mathrm{~Hz}$. This frequency could produce a beating effect with a $50 \mathrm{~Hz}$ imaging system, with a $36 \mathrm{~s}$ period. It was however possible to eliminate this lighting interference by employing the optical filters described above.

\section{Comparison of the Optical Volumetric Data to the Pneumatic 'Ground Truth'}

Performing an empirical least squares analysis yielded a calibration model with a precision of $\pm 7.5 \mathrm{~mL} / \mathrm{s}$ across the entire calibration range. To convert the NORM spirometer data back into physical flow rates, an inverse calibration model was required, compensating for systematic errors in the calibration flowmeter to yield high precision. These data successfully yielded an optical volume signal corresponding with the synchronously recorded pneumatic tidal flow. As expected, the volume signal led the flow signal with a phase difference of $90^{\circ}$ (i.e. given a flow 
rate of $d V / d t=\cos (\omega t)$, the volume signal will be $V=\sin (\omega t)+v_{0}, 90^{\circ}$ out of phase). Performing a numerical differentiation and noise filtering, yielded an optical flow signal in phase with the pneumatic flow signal, confirming the detection of tidal breathing.

The equipment shown in Figure 1 successfully captured data at 50fps, enabling 10fps of full 3D data to be recovered. The system was operated with the mannequin replaced by a volunteer patient breathing through a spirometer, while NORM captured vision data from their torso; an example of a captured image is shown in Figure 6.

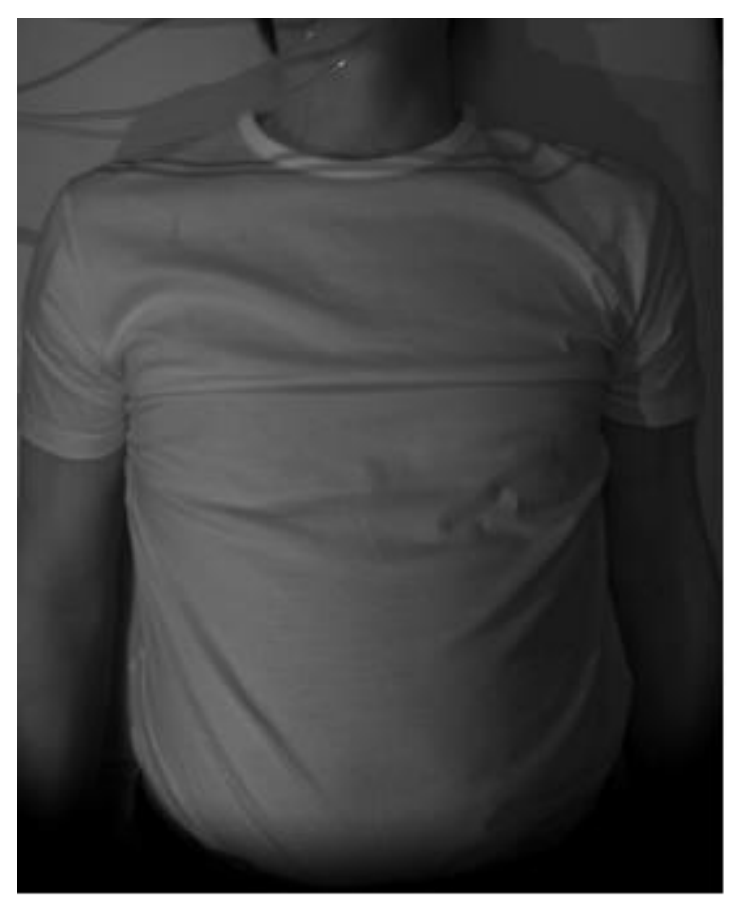

Figure 6. Example image from the NORM patient dataset.

Using the analysis model described above, the tidal breathing of the patient shown in Figure 6 was analysed. The resulting data are shown in Figure 7. A clear periodic signal is visible, of a 
frequency corresponding with that of the benchmark pneumotachograph data. There is also a consistent phase relationship between the two signals; which is as expected, with the pneumatic signal lagging behind the volume signal by $90^{\circ}$ (one quarter of a tidal cycle): i.e. the volume measurement peaks and troughs are around the areas of zero flow-rate, as would be expected. Both data in Figure 7 were recorded synchronously using the NORM system, so no manual time alignment was required or used to combine the two datasets. At seventeen seconds an occlusion is visible, which is believed to be caused by the patient swallowing. There is a corresponding plateau in the optical data with a slight delay as the phase relationship returns to its nominal relative value to the tidal flow rate.
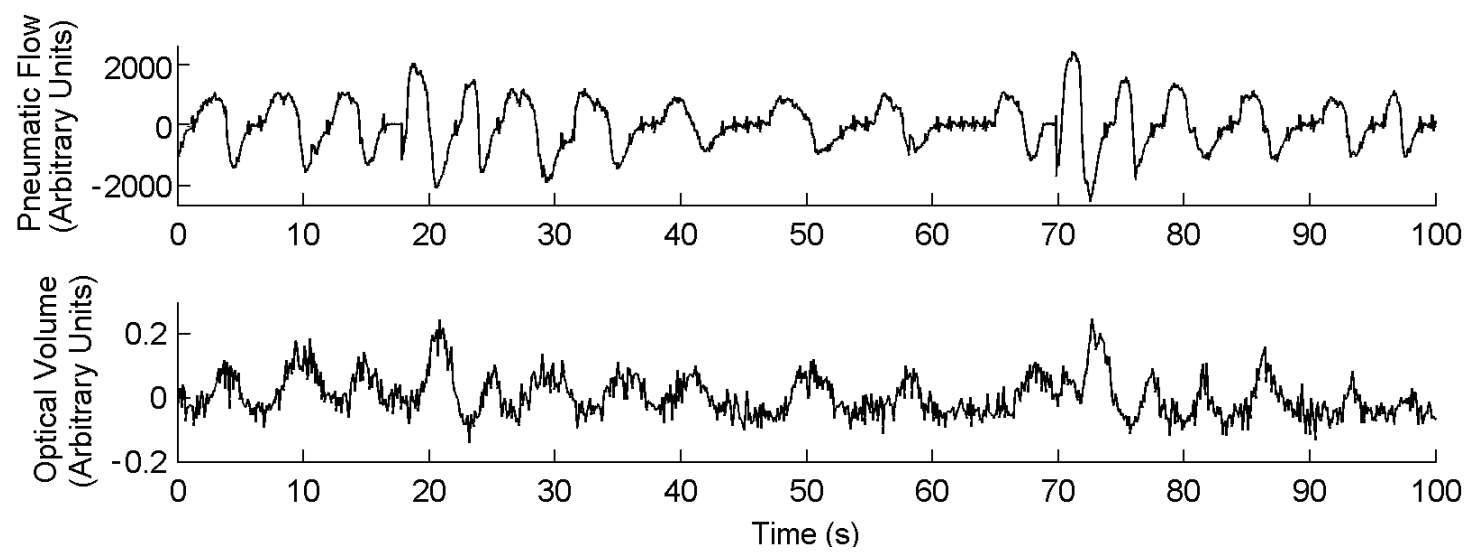

Figure 7. Concurrent plot of the time-dependent bulk volume measurement from the image data plotted against the synchronously captured benchmark pneumotachometer data. The pneumatic signal (dV/dt) lags the optical signal $(\mathrm{V}(\mathrm{t}))$ by $90^{\circ}$ (one quarter of a tidal cycle): i.e. the volume measurement peaks and troughs are around the areas of zero flow-rate.

The ambient lighting has been eliminated from the measurement by a combination of filtering and occlusion, and also the detected frequency here $\left(T_{\text {opt }} \approx T_{p n u} \approx 5 \mathrm{~s}\right)$ is well below the known 
lower limit of ambient interference $\left(T_{\min }=28.9 \mathrm{~s}\right)$; therefore it can be safely concluded that in Figure 7 the tidal breathing signal has been successfully detected. 


\section{DISCUSSION}

Breathing measurement represents a clinically useful technique for detecting and monitoring many medical conditions. However, many patients are either too young, too ill, or may lack the co-ordination required to perform conventional breathing measurement tests. This leads to delays in diagnoses and/or non-optimal treatments that are undesirable for the patients concerned and also a waste of money. For example it is desirable to be able to measure, rather than judge by symptoms, the extent of a child's response to medication that is intended to relieve wheeze. Detailed understanding of responses to drugs helps determine appropriate and tailored therapy, but is more difficult in those too young to undergo measures such as spirometry. There are, therefore, strong motivators for developing sensor systems able to monitor breathing for patients who are unable to utilize conventional (contact) breathing measurement devices. The NORM project focused on proof of principle of such a system, where an analysis model has been successfully developed and tidal breathing signals were detected (the calibration work has also produced new results useful for clinical spirometer calibration). NORM demonstrated that photometric stereo is suitable for breathing measurement for conscious adult patients in real time. However the intention has always been to further develop it for use with patients unable to cooperate, such as young children and those critically ill or unconscious, where its ability to work without patient cooperation and its unconstrained (i.e. it requires minimal or no environmental structuring) and unobtrusive mode of operation are expected to provide significant benefits. For example, since NORM employs non-visible light sources, breathing monitoring for sleeping patients can be achieved. The absence of any environmental structuring means that data can be acquired from patients with an unconstrained pose and position. This provides further opportunities for diagnosis of conditions such as Obstructive Sleep Apnoea 
(OSA), thereby potentially assisting with obviation of lengthy, laborious and expensive conventional polysomnography. The high spatial and temporal resolution that has been found to be achievable with the NORM approach means that it offers a capability to monitor or diagnose a number of respiratory related disorders that include neurological disorders, muscle motion and orthopaedic conditions; with the ability to detect regional movement providing further diagnostic functionality. Benefits may include increased sensitivity in detecting early signs of respiratory dysfunction in high risk patients, or localised diagnoses of particular lung conditions and identification of regions of dysfunction. The approach may also be useful for analysis of cardiac disorders and the monitoring of any conditions that lead to changes in torso morphology.

Although NORM successfully demonstrated the utility of dynamic photometric stereo in this application, further development work is needed for facilitating a system ready for employment in a clinical environment. Since the completion of NORM this work has continued through research at UWE in collaboration with NHS clinicians. The aim here is to further improve the photometric stereo approach to reduce noise and errors and thereby facilitate a robust system for non-contact pulmonary function testing (19). The focus of the work has also been extended to address diagnostics and possible appraisal of surgical interventions, with a particular emphasis on scoliosis. This condition, in the more severe forms, is generally associated with impaired pulmonary function, which in turn directly impacts on a patient's quality of life. Neurosurgeons, orthopaedic surgeons and respiratory clinicians have expressed interest in access to a non-contact system that could quickly and easily measure respiratory function before and after corrective spinal surgery. Studies have found that pulmonary function returns to preoperative levels 12-24 months following scoliosis correction, and a small improvement in forced expiratory volume has 
also been reported (20). The extent of the recovery also appears dependent upon the type of surgery employed, with endoscopic methods being reported as leading to greater long-term improvement in pulmonary function than open anterior approaches (21). The ability to detect and quantify these changes has motivated the focus of NORM on scoliosis, along with an appreciation that the technology can also be used to capture the morphology of the back of a patient in real time. This provides an opportunity to directly recover 3D torso features that can be used to measure the Cobb Angle, before and after surgery (22). This therefore has potential to offer a vision based screening or diagnostic functionality that offers benefits over the conventional approach of employing x-rays. These benefits include avoidance of the use of ionizing radiation, and potential for eliminating the error in measured Cobb Angle that can result from parallax effects when using orthogonal $\mathrm{x}$-rays to image a twisted spine.

Strengths of the NORM approach include its ability to work with unconstrained patients and in darkness. These factors will enable it to be used for sleep analysis and in critical healthcare applications such as monitoring patients in intensive and neonatal care. The technique does have some limitations; these include expected accuracy reductions when the patient is covered with thick bedclothes and possible occlusion or accuracy limits resulting from images and measurements being captured from only one direction. In fact we have found that improved measurement accuracy for breathing volume is obtained when two vision systems are used; one viewing the front and one the back, of a standing patient (19). We have also begun work on quantification of regional differences in chest wall motion that can be detected using the NORM spatial data. 
The ability of NORM to work for patients in bed and its relatively low-cost components that can be engineered into a device discretely mounted on the ceiling above the bed, means that it offers potential to be very widely employed in healthcare, or even potentially in a domestic setting. This has potential for intensive and neonatal care environments where such mounting is common, the low infection risk important and the patient may be relatively stationary for long periods. However, before this can occur, further development is needed to transform NORM from a technical proof-of-concept device into a robust prototype that has undergone extensive invivo testing, has MHRA approval and is ready for the ward. To summarise, NORM shows promise, but more work is needed to demonstrate that it can robustly and usefully monitor patient breathing in a wide range of real healthcare situations. However, if successful, NORM will offer a completely novel method of measuring volumetric breathing patterns during tidal respiration, in a variety of clinical situations where current methods are inapplicable. 


\section{ACKNOWLEDGEMENTS}

This paper presents independent research funded by the National Institute for Health

Research (NIHR) Invention for Innovation (i4i) Programme (II-FS-0908-10078). The views

expressed are those of the author(s) and not necessarily those of the NHS, the NIHR or the Department of Health.

The research associate who worked on the NORM project at UWE was Mr Scott Mandry.

\section{DISCLAIMER}

The views expressed are those of the author(s) and not necessarily those of the NHS, the NIHR or the Department of Health. 


\section{References}

1. Haland G, Carlsen K, Sandvik L. Reduced lung function at birth and the risk of asthma at 10 years of age. N Engl J Med. 2006;355:1682-1689.

2. Bisgaard H, Jensen S, Bønnelykke K. Interaction between asthma and lung function growth in early life. Am J Respir Crit Care Med 2012;185:1183-9.

3. Kozlowska W, Bush A, Wade A, Aurora P, Carr SB, Castle RA, Hoo AF, Lum S, Price J, Ranganathan S, Saunders C, Stanojevic S, Stroobant J, Wallis C, Stocks J; London Cystic Fibrosis Collaboration. Lung Function from Infancy to the Preschool Years after Clinical Diagnosis of Cystic Fibrosis. Am J Respir Crit Care Med 2008;178:42-49.

4. Izatt M, Harvey J, Adam C. Recovery of pulmonary function following endoscopic anterior scoliosis correction: Evaluation at 3, 6, 12, and 24 months after surgery. Spine 2006;31:24692477.

5. Fieselmann J, Hendryx M, Helms C. Respiratory rate predicts cardiopulmonary arrest for internal medicine inpatients. J Gen Intern Med 1993;8:354-360.

6. Subbe C, Davies R, Williams E, Rutherford P. Effect of introducing the Modified Early Warning score on clinical outcomes, cardio-pulmonary arrests and intensive care utilisation in acute medical admissions. Anaesthesia 2003;58:797-802. 
7. Goldhill D, McNarry A, Mandersloot G. A physiologically-based early warning score for ward patients: the association between score and outcome. Anaesthesia 2005;60:547-553.

8. Cretikos M, Chen J, Hillman K, Bellomo R. The objective medical emergency team activation criteria: a case-control study. Resuscitation 2007;73:62-72.

9. Hargrove, J, Zemper, E. Respiratory measurement ustilizing a novel laser displacement technique: normal tidal breathing. Biomedical instrumentation and technology 2009;43:327-331.

10. Tobin M, Laghi F. Monitoring of respiratory muscle function. In: Principles and practice of intensive care monitoring. New York, NY: McGraw Hill; 1998. p. 497-544.

11. Main E, Castle R, Stocks J, James I, Hatch D. The influence of endotracheal tube leak on the assessment of respiratory function in ventilated children. Intensive Care Medicine, 2001;27(11):1788-1797.

12. Peacock A, Morgan M, Gourlay S, et al. Optical mapping of the thoracoabdominal wall. Thorax 1984;39:93-100.

13. Layton A, Garber C, Basner R, et al. An assessment of pulmonary function testing and ventilatory kinematics by optoelectronic plethysmography. Clinical Physiology and Functional Imaging 2011;31:333-336. 
14. Brand, D. Lau, E. Cameron, J. Wareham, R. Usher-Smith, J. Bridge, P. Lasenby, J. Iles, R. Tidal breathing parameters measured by structured light plethysmography (SLP) and spirometry. American Thoracic Society International Conference Abstracts, 2010;B18.

15. Scalise, L. Ercoli, I. Marchionni, P. Tomasini, E. Measurement of respiration rate in preterm infants by laser Doppler vibrometry. Medical Measurements and Applications Proceedings (MeMeA), 2011 IEEE International Workshop.

16. Murthy, J. Pavlidis, I. Noncontact measurement of breathing function. Engineering in Medicine and Biology Magazine, IEEE. 2006;25(3):57-67.

17. Ranganathan S, Dezateux C, Bush A, et al. Airway function in infants newly diagnosed with cystic fibrosis. The Lancet 2001;358:1964-5.

18. Sun J, Smith M, Smith L, et al. Object Surface Recovery Using a Multi-Light Photometric Stereo Technique for non-Lambertian Surfaces Subject to Shadows and Specularities. Image and Vision Computing 2007;25:1050-1057.

19. Ahmad J, Sun J, Smith L, Smith M. An improved photometric stereo through distance estimation and light vector optimization from diffused maxima region. Pattern Recognition Letters Published online first: 19 September 2013. doi: http://dx.doi.org/10.1016/j.patrec.2013.09.005 
20. Newton, P.O. Parent, S. Marks, M. Prospective evaluation of 50 consecutive scoliosis patients treated with Thoracoscopic Anterior Instrumentation. Spine. 2005;S100-109.

21. Izzat M. Harvey J. Adam C, Fender D, Labrom R, Askin G. Recovery of pulmonary function following endoscopic anterior scoliosis correction: Evaluation at 3, 6, 12, and 24 months after surgery. Spine. 2006;31(21):2469-2477.

22. Herring J. Tachdjian's Pediatric Orthopaedics Volume $1.4^{\text {th }}$ ed. Saunders Elsevier, ISBN 9781416022213; 2008. p. 266. 


\section{Figure legends}

Figure 1. NORM Proof of Principle System; the final clinical system will either be mounted on the wall/ceiling, or will employ a simple bedside type stand.

Figure 2. Spectral response of Genie HM1400 / XDR Area Scan Monochrome Camera (Teledyne DALSA).

Figure 3: Emission spectrum of SFH 4730 Infrared LEDs.

Figure 4. Transmission Profile for Infra-Red filter Schneider SKR FIL 093, (profiles for two other filters are also shown).

Figure 5. Photometric stereo geometry for determination of the surface normal vector $\boldsymbol{n}$.

Figure 6. Example image from the NORM patient dataset.

Figure 7. Concurrent plot of the time-dependent bulk volume measurement from the image data plotted against the synchronously captured benchmark pneumotachometer data. The pneumatic signal (dV/dt) lags the optical signal $(\mathrm{V}(\mathrm{t}))$ by $90^{\circ}$ (one quarter of a tidal cycle): i.e. the volume measurement peaks \& troughs around the areas of zero flow-rate. 